\title{
LOS DESAFÍOS DE LA FORMACIÓN INICIAL DE PROFESORES DE MATEMÁTICAS COMO PROFESIONALES DEMOCRÁTICOS
}

\author{
Frederico da Silva Reis \\ frederico.reis@ufop.edu.br \\ https://orcid.org/0000-0001-6087-6483 \\ Universidade Federal de Ouro Preto (UFOP) \\ Ouro Preto, MG, Brasil \\ Kenneth Zeichner \\ kenzeich@uw.edu \\ https://orcid.org/0000-0003-0412-5575 \\ University of Washington (UW) \\ Seattle, WA, USA
}

Recibido: 29/12/2020 Aceptado: 01/02/2021

\begin{abstract}
Resumen
La formación inicial de profesores de Matemáticas en las universidades ha sido el centro de diversas investigaciones en el área de la Educación Matemática, que ha producido un gran número de estudios realizados a partir de supuestos teóricos y prácticos capaces de dar respuesta a las múltiples cuestiones que se le exigen al profesional docente, en el escenario educativo brasileño actual. Este artículo investiga las posibilidades y aportes de la formación inicial de los profesores de Matemáticas como profesionales democráticos, a partir de las ideas propuestas por el investigador Kenneth Zeichner. Es una investigación cualitativa con la investigación teórico-bibliográfica como principal componente metodológico. Los principales resultados obtenidos del análisis de la investigación discutida en este artículo señalan la existencia de varios y enormes desafíos en la formación de profesores de Matemáticas en las universidades bajo diferentes aspectos para la práctica del profesionalismo democrático, recomendándose, sin embargo, su inserción como política educativa en los programas de formación de profesores.
\end{abstract}

Palabras clave: Formación de Profesores; Profesionalismo Democrático; Educación Matemática.

\section{OS DESAFIOS DA FORMAÇÃO INICIAL DE PROFESSORES DE MATEMÁTICA COMO PROFISSIONAIS DEMOCRÁTICOS}

\begin{abstract}
Resumo
A formação inicial de professores de Matemática nas universidades tem sido o foco de diversas pesquisas na área de Educação Matemática, que tem produzido um grande número de pesquisas realizadas com base em pressupostos teóricos e práticos capazes de atender às múltiplas questões exigidas do profissional docente, no atual cenário educacional brasileiro. Este artigo investiga as possibilidades e as contribuições da formação inicial de professores de Matemática como profissionais democráticos, baseando-nos nas ideias propostas pelo pesquisador Kenneth Zeichner. Trata-se de uma investigação qualitativa tendo a pesquisa teórico-bibliográfica como principal componente metodológico. Os principais resultados obtidos das análises da pesquisa discutida neste artigo apontam para a existência de diversos e enormes desafios de se formar professores de Matemática nas universidades sob diferentes aspectos para a prática do
\end{abstract}


profissionalismo democrático, sendo recomendada, destarte, sua inserção como política educacional em programas de formação de professores.

Palavras chave: Formação de Professores; Profissionalismo Democrático; Educação Matemática.

\title{
THE CHALLENGES OF INITIAL EDUCATION OF MATHEMATICS TEACHERS AS DEMOCRATIC PROFESSIONALS
}

\begin{abstract}
The initial education of Mathematics teachers at universities has been the focus of several researches in the area of Mathematics Education, which has produced a large number of researches carried out based on theoretical and practical assumptions capable of meeting the multiple questions required of the teacher professional, in current Brazilian educational scenario. This article investigates the possibilities and contributions of the initial education of Mathematics teachers as democratic professionals, based on the ideas proposed by researcher Kenneth Zeichner. It is a qualitative investigation with theoretical-bibliographic research as the main methodological component. The main results obtained from the analysis of the research discussed in this article point to the existence of several and enormous challenges to prepare Mathematics teachers at universities under different aspects for the practice of democratic professionalism, being recommended, however, its insertion as educational policy in teacher education programs.
\end{abstract}

Keywords: Teacher Education; Democratic Professionalism; Mathematics Education.

\section{Introducción}

La sociedad mundial sufre constantes cambios debido a la evolución de las tecnologías y de la propia sociedad en su conjunto, en su forma de pensar y actuar. Ciertamente, tales cambios implican la necesidad de formar cada vez más profesores que estén dispuestos y sean capaces de comprender los complejos cambios que se han producido en los sistemas educativos $\mathrm{y}$, principalmente, en los procesos de enseñanza y aprendizaje.

Actualmente, el sector educativo requiere que sus principales actores estén atentos, ya sea hacia la educación integral del alumno para actuar como ciudadano en la sociedad cambiante, o hacia la formación inicial del profesor y, sobre todo, como es de nuestro interés, al futuro profesor de Matemáticas.

Gewirtz et al. (2009) afirman que los cambios sociales, culturales y también políticos han tenido implicaciones innegables para el trabajo del profesor, quien, a su vez, ha respondido de diferentes formas a los desafíos derivados de los cambios, y ese contexto, como no podía dejar de ser, engloba aspectos relacionados con la profesionalidad docente y, en profundidad, con la propia identidad profesional. 
Por un lado, Flores (2014, p. 853) traduce las identidades profesionales de los profesores en preguntas como "quiénes son, la imagen que tienen de sí mismos, los significados que atribuyen a su trabajo y a sí mismos como profesores, y los significados que otros les atribuyen”. La investigadora también señala que las identidades profesionales dependen de una variedad de factores, que van desde la forma en que los profesores se relacionan con sus alumnos, impregnando la disciplina que enseñan, los roles que desempeñan, los contextos en los que trabajan, desembocando a un "marco social y cultural más amplio en el que se inserta la docencia”, contemplando su biografía y oportunidades de desarrollo profesional.

Por otro lado, la investigación reciente en la formación del profesorado trae diferentes concepciones para el profesionalismo docente. Uno de los enfoques discutidos en diversos análisis de propuestas de formación de profesores que se han presentado es la centralidad de la idea de hacer de la docencia y la formación en sí, un trabajo más profesional. Según Zeichner (2019), a pesar de los intentos relativamente recientes de desregular y comercializar la educación $\mathrm{y}$, en consecuencia, de la propia formación de profesores, como señala Gastic (2014), se ha alcanzado un número significativo de logros en un esfuerzo por fortalecer el estatus profesional de la docencia y la formación del profesorado. En este contexto, el investigador afirma abogar por "un mayor enfoque en la preparación de los profesores como profesionales democráticos que se encuentran trabajando por las familias y comunidades de una manera respetuosa y genuinamente colaborativa, en lugar de tratar de 'salvar' a los alumnos de sus comunidades" (Zeichner, 2019, página 39, nuestra traducción).

Nuestro objetivo es presentar el profesionalismo democrático, concebido como una tendencia del profesionalismo docente y con base en el enfoque propuesto por el investigador Kenneth Zeichner, el segundo autor de este artículo, como una política pública fundamental para la formación inicial de profesores de Matemáticas, así como discutir algunos aspectos del contexto brasileño actual de esta formación, a la luz de algunos programas existentes en el ámbito de la relación universidad / escuela y las posibilidades que brindan a la práctica de formar futuros profesores de Matemáticas de la Educación Básica como profesionales democráticos.

Es, por lo tanto, el resultado de una investigación cualitativa, de carácter teórico y bibliográfico, realizada durante los estudios posdoctorales del primer autor del artículo, bajo la supervisión del segundo autor, en la Universidad de Washington (UW), en Seattle, WA, USA, en el primer semestre de 2020. 


\section{Algunas visiones sobre la formación inicial de los profesores de Matemáticas}

La necesidad de formar profesionales que puedan actuar para satisfacer las necesidades de la sociedad cambiante requiere que la formación del profesorado responda a sus demandas, especialmente en lo que se refiere a la capacidad de contribución, tanto directamente vinculada a su área específica de especialización como de manera más general, para la formación de un ciudadano capaz de actuar de manera transformadora y autónoma en el contexto socioeconómico-político en el que se inserta.

Adentrándonos en los documentos oficiales brasileños, inicialmente destacamos las Directrices Curriculares Nacionales para la Formación de Profesores de Educación Básica (2000) reafirmando el rol central de la formación inicial como preparación profesional que permite a los futuros profesores apropiarse del desarrollo de habilidades y también de autonomía tan fundamental para su desempeño en el escenario educativo actual. La formación inicial de los futuros profesores es necesaria para ofrecer bases sólidas de conocimientos que les permitan, en el ejercicio de su actividad docente, comprender la realidad del lugar donde trabajarán y, simultáneamente, reflexionar sobre su propia práctica docente. Una perspectiva que resume estas posibilidades combinada con la formación inicial nos la presenta Freitas (2004) al caracterizar al profesor que busca formarse:

Un profesional crítico reflexivo e involucrado en su propia formación, un profesional que se involucra políticamente en los procesos de toma de decisiones en diferentes contextos de su actuación - en el aula, escuela, asociaciones, etc., que dialoga con diferentes áreas de conocimiento y toma / utiliza el resultado de ésta como insumo para su desempeño en el aula y, en definitiva, ejerce y promueve la ciudadanía a partir de su propio desempeño (Freitas, 2004. p. 124, nuestra traducción).

Aún en esta perspectiva de la formación de un profesor que busca adecuar teorías y prácticas, posibilitando un cambio en la calidad de los procesos de enseñanza, pero además adecuándolos a la realidad en la que se inserta, las Directrices Curriculares Nacionales para la Formación de Profesores de Educación Básica (2000) señalan que:

Debemos afrontar el reto de hacer de la formación de profesores una formación profesional de alto nivel. Por formación profesional se entiende que no se trata de una formación genérica y no solo académica, sino que se centra en satisfacer las demandas de un ejercicio profesional concreto, ya que no basta con que un profesional tenga conocimientos 
sobre su trabajo. Es fundamental que sepa movilizar este conocimiento, transformándolo en acción (Brasil, 2000, p. 26, nuestra traducción).

Para ello, es fundamental pensar que el profesor debe estar formado inicialmente para comprometerse a intervenir en la realidad de sus alumnos. Sin embargo, esto solo será posible si los cursos de formación inicial de profesores desarrollan planes de estudio capaces de dotar a los futuros profesores de un conjunto de competencias no tan alejadas de la realidad educativa. Pimenta (1999) nos recuerda que los contenidos y actividades alejados de la realidad y la práctica social real de educar, poco contribuyen a conformar una nueva identidad para el profesional docente.

La formación inicial del profesor, en este contexto, debe asumir supuestos teóricos y prácticos capaces de responder a las múltiples cuestiones planteadas en el actual escenario educativo brasileño. En concreto, en la perspectiva de la formación inicial del profesor de Matemáticas, Perrenoud (2001) destaca la importancia de que el futuro profesor no sea un simple ejecutor de tareas listas y terminadas, de hecho, ya que la propia Matemática fue concebida durante mucho tiempo como ciencia lista y terminada, sino más bien para convertirse en un profesional capaz de conocer y buscar nuevas posibilidades en los procesos de enseñanza de las Matemáticas. Así, la formación inicial de los profesores para enseñar Matemáticas no solo requiere una formación de carácter estrictamente académico, con un enfoque únicamente en conocimientos matemáticos específicos, sino que es necesario pensar en una formación que pueda atender las diversas demandas de la sociedad y, así, las demandas pedagógicas correlacionadas.

Considerando la formación inicial del profesor de Matemáticas que se desarrolla en los cursos de Licenciatura en Matemáticas que ofrecen las universidades, cabe destacar la relevancia del espacio de tiempo destinado a la formación inicial del profesor, ya que es durante este período que el futuro profesor desarrollará los conocimientos y habilidades necesarios para actuar en la docencia con profesionalismo y autonomía. Es también en este período que el futuro educador matemático podrá percibirse como un agente de transformación de la sociedad, actuando activamente por los cambios necesarios en los procesos de enseñanza y aprendizaje de las Matemáticas, como lo destacan las Directrices Curriculares Nacionales para Cursos de Matemáticas, Bachillerato y Licenciatura (2001):

Se destaca que el educador matemático debe ser capaz de tomar decisiones, reflexionar sobre su práctica y ser creativo en la acción pedagógica, reconociendo la realidad en la que se inserta. Más que eso, 
debe avanzar hacia una visión de que la acción práctica genera conocimiento (Brasil, 2001, p. 26, nuestra traducción).

En el actual contexto brasileño de formación de profesores de Matemáticas, es necesario que la formación inicial permita al futuro profesor experimentar los ambientes escolares, incluso antes de la conclusión de su graduación, a través de prácticas supervisadas (pasantías en escuelas) obligatorias, según lo prescrito por la Ley 11.788/2008. El objetivo es posibilitar una experiencia sumamente importante para el proceso de formación del futuro profesor de Matemáticas, que le permita conocer la realidad educativa de las escuelas de los distintos sistemas de enseñanza existentes en su geolocalización. La práctica supervisada es, por lo tanto, una forma de integrar el conocimiento académico con el conocimiento escolar y, a partir de esta integración, brindar una oportunidad de acercarse a su realidad profesional como parte del proceso de formación del futuro profesor de Matemáticas.

Las Directrices Curriculares Nacionales para Cursos de Matemáticas, Bachillerato y Licenciatura (2001) también mencionan las prácticas docentes obligatorias, concebidas de manera fundamentalmente complementaria a los desarrollos teóricos inherentes a la formación inicial del profesor de Matemáticas:

La práctica es la forma misma de hacer las cosas, cuyo contenido está atravesado por una teoría. Así, la realidad es un movimiento constituido por la práctica y la teoría como momentos de un deber más amplio, a medida que la práctica es el momento en el que se busca hacer algo, producir algo y que la teoría busca conceptualizar, significar y así gestionar el campo y el significado de esta actuación (Brasil, 2001, p. 14 , nuestra traducción).

Así, nos dimos cuenta de que la práctica supervisada tiene como objetivo concatenar la teoría y la práctica docente, teniendo como uno de sus objetivos brindar al futuro profesor de Matemáticas la oportunidad de seguir diversos aspectos de la vida escolar más allá de los muros de las aulas universitarias. En teoría, tal oportunidad debería ser muy útil para coincidir con Contreras (2002), para quien el profesor debe presentar las siguientes características, a las que él llama profesionalidad, que ha de ser construida a lo largo de su formación inicial:

- Obligación moral - teniendo como componentes la preocupación por el bienestar de los alumnos y la ética, abarcando las relaciones de afectividad y motivación;

- Compromiso con la comunidad - estableciendo, inicialmente, con los profesores $\mathrm{y}$, posteriormente, con la sociedad en su conjunto, la intervención en los problemas sociales y políticos, y entendiendo la escuela como lugar de preparación para la vida futura, como agente regulador de la sociedad (libertad, igualdad, justicia); 
- Competencia profesional - trasciende el dominio de habilidades y técnicas y surge de la interacción entre obligación moral y compromiso con la comunidad (Contreras, 2002, p. 73, nuestra traducción).

También nos corresponde a nosotros evaluar si las prácticas supervisadas y las prácticas docentes han podido, de hecho, cumplir con su papel de proporcionar una integración real entre profesor y escuela y entre teoría y práctica de forma dialéctica y reflexiva, con el objetivo de no sólo cumplir las cargas obligatorias en el plan de estudios de los cursos de Licenciatura en Matemáticas, sino también proporcionar al futuro profesor la construcción de una rica gama de conocimientos prácticos necesarios para el ejercicio de su docencia.

En este sentido, parece importante traer a la discusión sobre algunas cuestiones planteadas en este panorama actual de la formación inicial del profesor de Matemáticas para la Educación Básica, la necesidad de repensar su propio significado y el papel de su acción como profesional docente.

\section{Algunas visiones sobre el profesionalismo docente}

No es tarea fácil en la literatura disponible, llegar a una definición razonablemente consensuada de lo que es el profesionalismo docente y sus relaciones intrínsecas o extrínsecas con temas como la profesionalización docente y la profesionalidad docente que, por no ser nuestro foco, no trataremos en este artículo.

En general, podemos pensar que el profesionalismo docente concierne a la naturaleza y calidad de todo trabajo docente en su práctica. Buscando asociar la práctica docente con diferentes contextos, Sockett (1993) afirma que el profesionalismo docente puede manifestarse bajo diferentes dimensiones.

La primera dimensión del profesionalismo docente se da en las comunidades profesionales, a partir de las relaciones que desarrollan los profesores en las instituciones y, más específicamente, en los departamentos en los que trabajan. Una segunda dimensión está relacionada con los conocimientos específicos, sobre los que los profesores traen consigo visiones y creencias. La tercera dimensión es lo que el investigador describe como una rendición de cuentas profesional, identificada con una obligación moral que tienen los profesores hacia sus alumnos y el público en general. Finalmente, la cuarta dimensión del profesionalismo docente es vista como un ideal de servicio profesional relacionado con un propósito moral de la 
docencia que también puede asociarse con un compromiso que asumen los profesores en contribuir al bienestar y desarrollo de sus alumnos.

A nuestro juicio, estas dimensiones del profesionalismo docente, si bien impregnan diversos contextos prácticos del trabajo docente, aún la sitúan a partir de relaciones y actitudes derivadas básicamente de concepciones manifestadas por los profesores, derivadas de su experiencia institucional / profesional o de su moral / ética, sin mirar otros posibles contextos para el trabajo docente, como el ambiente colaborativo que se puede establecer en el trabajo de los profesores con las comunidades escolares, que generalmente se conciben de una manera más estricta, considerando solo a sus actores tradicionales como alumnos y profesores, pero que en este trabajo serán concebidas de una manera más amplia, considerando también a otros actores hasta ahora considerados, como mucho, agentes secundarios en el escenario escolar, como las familias de los alumnos e incluso las asociaciones comunitarias y los movimientos sociales locales.

En esta perspectiva, Hargreaves (1994, p. 424) apuntó a uno nuevo profesionalismo docente que, además del desarrollo profesional y de las relaciones institucionales, puede concebirse como un movimiento "más allá de la autoridad tradicional y la autonomía del profesor en el sentido de nuevas formas de relación entre colegas, alumnos y padres". Según el investigador, estas relaciones deben ser más estrechas y colaborativas, ya que implican una "negociación más explícita de roles y responsabilidades".

En su análisis de los discursos del profesionalismo docente, Flores (2014) afirma que este movimiento:

[...] resulta de extender el trabajo de los profesores más allá del aula para involucrar a toda la escuela en la que las prioridades educativas, la planificación e implementación del currículo y los problemas de progresión y continuidad de los alumnos requieren una mayor coordinación por parte de los profesores. Es decir, el nuevo profesionalismo, frente al antiguo profesionalismo, incluye, entre otros aspectos, un paso del individualismo a la colaboración, de las jerarquías a los equipos, del proceso al producto, de la autoridad al contrato y de la supervivencia a la emancipación, aunque esta perspectiva es cuestionada por otros autores (Flores, 2014, p. 859, nuestra traducción).

Observamos que las preguntas sobre esta nueva perspectiva del profesionalismo docente pueden estar relacionadas con varias implicaciones para la práctica docente que conlleva tal cambio de paradigmas. Sí, porque, fundamentalmente, se trata de un cambio de paradigmas, especialmente los que sitúan al profesor en el epicentro educativo, a partir de su papel 
centralizador / catalizador de las prioridades escolares. Como explica la investigadora, la autoridad del profesor, fuertemente reforzada a lo largo de su formación, da paso ahora a una construcción colectiva de saberes y prácticas, que no siempre es fácil de aceptar o comprender.

Así, podemos ver que los cambios en las concepciones del profesionalismo docente han acompañado no solo a los cambios característicos de los entornos escolares, sino también a las transformaciones organizativas en el propio mundo del trabajo. En este contexto, Sachs (2003) propone una oposición entre el profesionalismo gerencial y el profesionalismo democrático.

El profesionalismo gerencial surge teniendo en cuenta algunos de los principios ligados al gerencialismo, como la primacía de la gestión eficiente y autónoma, sustentada en la búsqueda de la efectividad de los resultados. Según la investigadora, los reflejos de la transposición de estos principios al contexto escolar inciden en el trabajo docente cuando pensamos en la burocracia en la docencia, y en las prácticas de gestión escolar que, peligrosamente, pueden transitar el camino de los "resultados medibles" que conducen a rankings, especialmente considerando la rendición de cuentas por parte de los profesores, realizada de manera comparativa con metas preestablecidas, a menudo por agencias estatales o federales externas. Sachs (2003) destaca que, en esta perspectiva, el "buen profesional" es aquel que trabaja de manera eficiente y eficaz, con el fin de cumplir con los criterios estandarizados que se han establecido para profesores, alumnos e, institucionalmente, también para las escuelas.

Entendemos que esta visión del profesor como un profesional que tiene la obligación de cumplir con los criterios preestablecidos y que lo coloca, en cierto modo, en "pie de igualdad" con los profesionales de la mayoría de los sectores de la sociedad actual, corrobora una visión del mundo apoyada en los principios de competitividad, control y regulación que rigen el mundo capitalista en el que, innegable e inevitablemente, estamos todos insertados. Además, el hecho de que estemos insertados en un mundo regido por ideales gerenciales no nos impide concebir otras formas de trabajo docente que transgredan, en la medida de lo posible, el status quo subyacente.

En este sentido, Sachs (2003) presenta el profesionalismo democrático como capaz de “desmitificar el trabajo profesional”, precisamente porque apunta a construir alianzas entre profesores y otros agentes, a partir de una acción colaborativa y cooperativa. Esta desmitificación del desempeño profesional provoca un choque en las estructuras escolares tradicionales, según Flores (2014), ya que nuevamente el profesor: 
[...] viene con responsabilidades más amplias, más allá del aula, incluyendo la contribución a la escuela, al sistema educativo, a la comunidad y a los alumnos, así como responsabilidades colectivas como profesión, con vistas a construir una sociedad más justa y democrática mediante, por ejemplo, la investigación, la innovación, etc. (Flores, 2014, p. 860, nuestra traducción).

Es interesante observar que, tanto el nuevo profesionalismo docente como el profesionalismo democrático, atribuyen responsabilidades a los profesores "más allá del aula", lo que puede parecer paradójico con el hecho de que, al menos teóricamente, una postura de subordinación a los principios gerenciales es que debería acarrear un mayor "esfuerzo profesional" en el intento, a veces sin gloria, de lograr metas comparativas.

Es necesario traer a esta discusión algunos valores que Sachs (2000) identifica como fundamentos de un enfoque responsable del profesionalismo docente: Aprendizaje (profesores como aprendices, individualmente y con sus colegas y alumnos); Participación (profesores como sujetos activos de su propia realidad profesional); Colaboración (profesores como promotores de la colegialidad dentro y entre comunidades escolares); Cooperación (profesores como desarrolladores de lenguajes y tecnologías para la documentación y discusión de sus prácticas); Activismo (profesores como agentes públicos en temas relacionados con la educación y la escolarización).

Así, consideramos imprescindible considerar tales fundamentos en la concepción de un profesionalismo democrático que incentiva la proactividad en el desempeño de los profesores, frente a la deseable pro receptividad en el ámbito de actuación de un profesional docente eficiente o, por así decirlo, un “docente profesional”.

\section{Una mirada diferente sobre el profesionalismo democrático}

Entre las concepciones de profesionalismo democrático abordadas en estudios sobre profesionalismo docente, queremos destacar el aporte de las ideas traídas a la discusión por el investigador norteamericano Kenneth Zeichner.

Defendiendo la importancia de materializar el potencial democrático de la educación pública y la formación de profesores, Zeichner (2019) sostiene que el profesionalismo democrático ofrece una forma de contribuir a la formación de profesores con el fin de volver a discutir las posibles relaciones entre las escuelas públicas, las comunidades minoritarias y los programas de formación de profesores: 
Este enfoque decolonial de la formación de profesores que trata activamente de beneficiarse de la experiencia en las comunidades locales minoritarias, busca 'romper' las jerarquías de poder y conocimiento existentes y crea la base para nuevas alianzas entre profesores, sindicatos de profesores, formadores de profesores y movimientos sociales en comunidades marginadas que buscan un rol activo en la transformación de sus propias comunidades. El resultado es una nueva estructura híbrida para los programas de formación de profesores que moldean la visión emancipadora que, a menudo, es articulada por programas, pero no practicada (Zeichner, 2019, p. 38, nuestra traducción).

Inicialmente, el investigador permea la "agenda de profesionalización" en la formación de profesores en Estados Unidos (Zeichner, 2009), destacando como una discusión importante que surge de ella, una amplia distinción entre preparar a los profesores como profesionales y preparar a los profesores como burócratas o técnicos (Zeichner, 2016). El autor reconoce los aportes de esta comparación entre preparar a los profesores como profesionales reflexivos y técnicamente competentes, capaces de aprender en y desde sus prácticas de aula, y preparar a los profesores como técnicos estrictamente capacitados para implementar fielmente prácticas de enseñanza con precisión que se cree que promueven alguna visión del aprendizaje del alumno.

Todavía, Zeichner (2019) afirma que este tipo de "comparación amplia” también puede ser engañosa. Entre las razones dadas para justificar tal afirmación, el investigador destaca la variación existente en las concepciones de la práctica docente, trayendo la representación del continuum en la formación de profesores descrito por Feiman-Nemser (2012). En un extremo del continuum, está el enfoque tecnológico asociado con un modelo de formación de "aprender a enseñar", cuyo enfoque es enseñar a los profesores en formación a utilizar un conjunto de habilidades y prácticas con competencia y fieldad para apoyar el aprendizaje de los alumnos. En el otro extremo, está el enfoque de orientación deliberativa para la práctica de las "habilidades técnicas", cuyo enfoque es formar profesores como tomadores de decisiones técnicamente competentes, y que también deben estar preparados para adaptar reflexivamente las prácticas docentes y ejercer su juicio sobre el uso de estas prácticas, en momentos y circunstancias particulares.

Sin embargo, Zeichner (2019) señala que el simple hecho de afirmar que se está preparando a los profesores para ser técnicamente competentes o para ser reflexivos sobre su enseñanza dice "muy poco sobre la naturaleza de su preparación", porque la reflexión del profesor se ha utilizado para apoyar tanto una visión tecnológica como una visión deliberativa 
más expansiva de la práctica profesional: “Al final, los profesores deben estar preparados para ser técnicamente competentes y reflexionar sobre su práctica, pero la naturaleza y la calidad de su preparación pueden variar ampliamente, dependiendo de cómo estos conceptos están definidos e implementados" (Zeichner, 2019, p. 39, nuestra traducción).

Zeichner (2019) también señala la existencia de diferentes visiones sobre el profesionalismo en la enseñanza y, tras discutir las características y contextos de las principales concepciones del profesionalismo docente, presenta pilares del profesionalismo democrático tal como lo conciben internacionalmente varios académicos. Dzur (2008), por ejemplo, defiende una participación pública más amplia en la deliberación sobre los principales temas sociales dentro y fuera del ámbito escolar, pero señala que "esto no disminuye la autoridad, status y responsabilidad profesional" de los profesores. Asimismo, Sachs (2001) defiende la acción cooperativa entre profesores y otros actores de la educación, como alumnos, padres y miembros de la comunidad "en cuyo nombre se toman tradicionalmente las decisiones".

Cabe hacer una mención especial al espíritu pionero de las ideas de Cuban (1969), quien criticó el hecho de que el profesionalismo fuera utilizado como un "código" para mantener a los padres alejados del ambiente escolar y como una forma de resistir el contacto "cara a cara" significativo entre escuelas y padres y entre profesores y comunidades. Desde entonces, ha instado a los profesores a "ampliar su visión", incluida su participación activa con los padres y su participación en la vida comunitaria, y argumentó que los programas de formación de profesores deberían prepararlos para esto.

Zeichner (2019, p. 41, nuestra traducción), entonces, afirma que "la idea de profesionalismo democrático centra a los alumnos, familias y comunidades en el trabajo de los profesores y en los programas de formación de profesores", constituyendo así una alternativa con "potencialidad de ayudar a gestionar productivamente las tensiones y contradicciones que existen desde hace mucho tiempo entre los educadores profesionales y las comunidades no dominantes a las que deben servir".

Sin embargo, es necesario considerar las dificultades y complejidades de la formación de profesores como profesionales democráticos. Aunque señala un cambio significativo en la formación inicial de profesores en Estados Unidos, Zeichner (2018) afirma que los programas de formación tradicionales no pudieron cambiar las estructuras de poder y conocimiento de una forma que apoye e incluya la experiencia y las perspectivas que existen dentro de las 
comunidades no dominantes y, en este contexto, el investigador defiende una forma de profesionalismo en las escuelas y en programas de formación de profesores en la que:

[...] las jerarquías de poder actuales se reducen y donde profesores, sindicatos de profesores y formadores de profesores trabajan, de manera más genuina, formas colaborativas con las familias y comunidades en las escuelas, en los programas de formación de profesores y en las comunidades. Hoy en día, la mayoría de los profesores de colegios y universidades, con base en escuelas y educadores independientes en los Estados Unidos, dicen que sus programas están preparando a los profesores para la 'justicia social'. Creo que el profesionalismo democrático es una parte esencial de cualquier enfoque de la formación de profesores que clama por el manto de justicia social (Zeichner, 2019, p. 42, nuestra traducción).

Como forma de justificar mejor sus creencias y, al mismo tiempo, acentuar sus críticas, Zeichner (2019) concluye afirmando que la mayoría de los programas de formación de profesores, incluidos los que pretenden reivindicar la justicia social, han preparado a los profesores que no se han "beneficiado del conocimiento y de la expertise que existe en las familias y comunidades de sus alumnos" y que continúan tratando de "salvar" a los alumnos de sus comunidades. Entendemos que la idea de "salvar alumnos" se puede interpretar de diferentes formas. Una posible interpretación es una postura jerárquica del conocimiento académico, traído y representado por profesores formados en las universidades, capaz de modificar / mejorar las condiciones en las que viven los alumnos, "salvándolos así de sus comunidades". Reanudaremos esta discusión en las conclusiones de este artículo.

Finalmente, antes de buscar una costura entre el profesionalismo democrático y la formación inicial de los profesores de Matemáticas, eje central de este trabajo, hay que destacar la advertencia de Zeichner (2019), a la que debemos prestar atención, especialmente en el contexto social-político-económico actual brasileño y mundial, dentro del cual el rol de las escuelas y universidades, como locus de formación inicial y continuada de profesores y también el rol de los propios formación de profesores ha sido ampliamente cuestionado por la sociedad en general:

El profesionalismo democrático en la enseñanza y en la formación de profesores puede contribuir potencialmente tanto para la justicia educativa como para el bienestar de la comunidad, pero debido a las presiones actuales sobre las escuelas y las instituciones de formación de profesores, existe el peligro de que se transforme en una moda benigna como el slogan de 'asociaciones' que no van a contribuir para cambiar las jerarquías de poder existentes y para conectar las escuelas y los 
programas de formación de profesores más estrechamente con las comunidades a las que supuestamente deben servir y ante las cuales deben rendir cuentas (Zeichner, 2019, p. 41, nuestra traducción).

\section{Desafíos para formar a los profesores de Matemáticas como profesionales democráticos}

Formar a los profesores de Matemáticas como profesionales democráticos conlleva responsabilidades que deben asumirse colectivamente con mayor densidad que las que exige el principio de formación de profesores simplemente como profesionales "competitivos para el mercado de trabajo".

Es necesario vislumbrar, entonces, cambios que impacten el escenario actual de la formación inicial del profesor de Matemáticas, desde la perspectiva de un profesor que comprende y asume su rol de intervenir en la realidad, tanto la realidad de las escuelas en las que actuará como las comunidades en las que ellas están insertadas.

Entendemos, además, que los cambios exigen avanzar en temas relacionados con la realidad de la formación inicial de los profesores de Matemáticas en las universidades brasileñas que, por ahora, se presentan como enormes desafíos a superar, ya que ciertamente implican un cambio de creencias, relaciones y políticas.

El primer reto a afrontar sigue siendo superar la creencia de que para formar un buen profesor de Matemáticas, no sólo es necesario, como a la vez suficiente, priorizar conocimientos matemáticos específicos que, a pesar de ser fundamentales en el proceso de formación, no minimizan la función y la importancia de conocimientos y saberes de otra índole en este complejo proceso. Como muy bien señalan Born, Prado y Felippe (2019):

Esta formación específica en contenidos, sin embargo, debe articularse sistemáticamente con la enseñanza, lo que requiere que los futuros profesores tengan, en su formación académica, experiencias vinculadas al proceso de escolarización, enseñanza y aprendizaje. La formación y el conocimiento sobre las estructuras y materiales educativos son fundamentales para que el profesor se familiarice con el territorio docente, como instituciones, organizaciones, contextos y currículos (Born, Prado \& Felippe, 2019, p. 4, nuestra traducción).

Las investigadoras también argumentan que la integración de todos estos aspectos de la formación docente sólo puede darse con la inserción permanente de la práctica durante la formación de profesores y que la capacidad de articular "conocimientos teóricos con elementos de la práctica" está en la reflexión por parte de los profesores sobre el trabajo docente en el aula. 
En esta perspectiva, Born, Prado y Felippe (2019) destacan la importancia de que los futuros profesores se inserten en las escuelas a través de prácticas supervisadas, obligatorias en los cursos de formación inicial de profesores, las cuales deben estar "rodeadas de artefactos de la práctica, como los estudios de caso y actividades de los alumnos".

Como señalamos anteriormente, la práctica supervisada debería, como probablemente se prevé en el Proyecto Pedagógico de Curso (PPC) en muchas instituciones brasileñas, brindar al futuro profesor de Matemáticas la oportunidad de seguir varios aspectos de la vida escolar que le esperan. Sin embargo, para que este futuro profesor pueda entrar en el momento de la práctica supervisada para asumir, en su futura práctica docente, el compromiso con la comunidad escolar, es fundamental que se presente en esta comunidad no como un poseedor de conocimientos matemáticos "estrictamente construidos en la universidad" y que sólo está ahí para compartir parte de su rico conocimiento teórico con los alumnos, de hecho, una postura que está en línea con muchos profesores que, quizás incluso inconscientemente, están tratando de "salvar" a sus alumnos.

Formar a los profesores de Matemáticas como profesionales democráticos implica la valoración de las prácticas supervisadas como momentos esenciales para el inicio de la construcción de la profesionalidad docente (Contreras, 2002), especialmente en el aspecto de la competencia profesional, que trasciende el dominio de conocimientos, habilidades y técnicas y no debe estar desvinculado del compromiso con la comunidad escolar.

Otro gran desafío a enfrentar es la apreciación de la relación entre universidades y escuelas, que innegablemente se está consolidando de manera innovadora y rápida, especialmente considerando los últimos años, en los que se han implementado varios programas públicos directamente vinculados a la formación de profesores.

Tanto en el escenario nacional brasileño (Fiorentini, 2009) como en el escenario internacional (Vargas, 2017), la relación universidad / escuela ha sido objeto de investigaciones producidas con un enfoque en las posibilidades que esta relación aporta a la formación de profesores, particularmente por parte de la constitución de ambientes donde se puedan construir prácticas de colaboración, reflexión e investigación.

Así, Cyrino (2013) destaca la importancia de incluir las posibilidades que surgieron de la relación entre universidades y escuelas en el contexto de la formación inicial de los profesores de Matemáticas. La autora investiga la formación inicial y continuada de profesores que enseñan 
Matemáticas en comunidades de práctica, concebidas como grupos formados por profesoresinvestigadores, futuros profesores y profesores de Matemáticas que estudian las prácticas escolares y entienden las escuelas como importantes espacios de formación. Argumentando que las comunidades de práctica han demostrado ser un espacio fructífero para explorar los procesos de aprendizaje para profesores y futuros profesores que enseñan Matemáticas, Cyrino (2013) afirma:

Entendemos que las propuestas de formación que incluyan la valoración de experiencias, repertorios y conocimientos, y que también permitan la asunción de sus aprendizajes, a través de la negociación de significados, permeadas por factores como el respeto, la confianza, el desafío y la solidaridad, parecen más adecuadas al proceso de formación de profesores (Cyrino, 2013, p. 5205, nuestra traducción).

Ciertamente, la relación universidad / escuela ha revelado su potencial para valorar experiencias, repertorios y conocimientos adecuados a la formación inicial de los profesores de Matemáticas, especialmente cuando reconocemos la contribución de los programas creados por el gobierno brasileño, basados en acciones políticas educativas como por ejemplo, el Programa Institucional de Becas de Iniciación Docente (PIBID) y el Programa de Residencia Pedagógica (PRP).

No es el foco primario del presente estudio investigar el aporte antedicho, todavía, cabe destacar la investigación realizada por Santana y Barbosa (2019), que específicamente analizó y problematizó cómo se da la relación universidad / escuela en el Programa Residencia Pedagógica, basado en estrategias de poder y modos de subjetivación puestos en funcionamiento por los discursos, tomando como referencia grandes "ejes de experiencia", como el conocimiento del contexto y la cultura de la escuela, el conocimiento de la gestión del sistema de enseñanza, de la escuela y del aula, la experimentación de técnicas, didácticas y metodologías de enseñanza y, finalmente, la asociación entre práctica supervisada, Programa de Residencia Pedagógica y escuela rural.

Destacando que la interacción entre los diferentes campos formativos (universidad / escuela), y promoviendo un proceso cíclico de aprendizaje, los cambios y transformaciones en los sujetos, contribuyen a la constitución del profesor de Matemáticas, y los investigadores concluyen:

Los resultados indican que los modos de subjetivación característicos de este momento de formación lo inclinaron hacia la hermenéutica colaborativa. La aproximación entre universidad y escuela permitió 
reflexionar sobre el conjunto de prácticas que se establecen en el partenariado entre las instituciones y entre los diferentes sujetos que contribuyen a la formación del profesor que enseña Matemáticas, en línea con los estudios en el área de Educación Matemática (Santana \& Barbosa, 2019, p. 21, nuestra traducción).

Esta conclusión tiene implicaciones para nuestra discusión sobre la formación de profesores de Matemáticas. Al igual que en la práctica supervisada, el "encuentro" entre la universidad y la escuela posibilitado por programas como PIBID y PRP puede y debe ser visto como un espacio privilegiado de experimentación y, en esta perspectiva, se requiere un esfuerzo institucional conjunto para que más y más futuros profesores reconozcan y valoren este espacio, involucrándose en estos programas, a partir de su formación inicial en las universidades y, como reflejo de este aprecio, actuando como preceptores en las escuelas involucradas, lo que también puede contribuir a su formación continuada.

Las tensiones ahora planteadas en la aproximación de la universidad con la escuela y de los futuros profesores con profesores actuantes, pueden posibilitar la construcción de nuevas prácticas emergentes y, al contribuir al fortalecimiento del respeto mutuo entre la universidad y la escuela, también amplía los compromisos de los profesores de Matemáticas, futuros y actuantes, hacia la escuela y su comunidad.

Formar a los profesores de Matemáticas como profesionales democráticos pasa por el reconocimiento de las escuelas como instancias centrales en la construcción de la profesionalidad docente (Contreras, 2002), especialmente en el aspecto de consolidar el compromiso con la comunidad escolar, tanto el compromiso institucional por parte de la universidad, como el compromiso personal por parte de los profesores.

Finalmente, un desafío mayor a enfrentar es la discusión de políticas públicas de formación de profesores que legitimen a la escuela como locus de formación y a la comunidad escolar como propositiva, y no solo como una colectividad sumisa a las resoluciones provenientes de los organismos gubernamentales.

En el contexto educativo brasileño, los discursos sobre la importancia de la "calidad de la educación ofrecida por la escuela básica" y de la "calificación de la mano de obra para el mercado de trabajo" se han entrelazado, en cierta medida, con la discusión sobre la formación de profesores, en las acciones y políticas propuestas por el Ministerio de Educación brasileño, según lo informado por Barbosa y Fernandes (2017): 
En el contexto de las transformaciones sociales, políticas y económicas de las últimas décadas, específicamente en los años posteriores al proceso de redemocratización en Brasil, la formación de profesores cobró protagonismo en el ámbito de las políticas educativas, como consecuencia de los debates que ganaron fuerza sobre la calidad de la enseñanza ofrecida en la escuela básica. Es decir, como tales discusiones han ocurrido también en el contexto de importantes demandas de una educación básica que responda a los requerimientos económicos relacionados con la mano de obra cualificada para el mercado de trabajo contemporáneo, la escuela ha pasado a ser vista como la responsable de crear condiciones para que los individuos desarrollen sus habilidades y aprendan los contenidos necesarios para comprender y participar en las relaciones sociales, económicas y culturales cada vez más plurales (Barbosa \& Fernandes, 2017, p. 25, nuestra traducción).

Si bien consideramos el perfil idiosincrásico del reciente escenario político-educativo brasileño realizado por las investigadoras, la imagen de una escuela responsable por crear las condiciones para la formación de una mano de obra "cualificada" para el mercado de trabajo, cumpliendo con “estándares de calidad” de la enseñanza, parece indicar que los profesores de esa escuela deben cumplir con criterios también estandarizados, a fin de lograr efectividad en cuanto al desarrollo de habilidades y el aprendizaje de los contenidos necesarios por parte de sus alumnos; es decir, entendemos que, desde esta perspectiva, los profesores deben formarse como profesionales gerenciales.

Sin embargo, varias investigaciones en Educación Matemática (Franchi \& Reis, 2012; Rosa, Reis \& Orey, 2012; Nacarato \& Paiva, 2017; Cyrino, 2018; Reis \& Esteves, 2020) recomiendan que la formación inicial de los profesores de Matemáticas sea un espacio que refuerze el compromiso del profesor con sus alumnos mucho más allá de los procesos de enseñanza y aprendizaje, pero también estime la importancia del trabajo docente para el crecimiento de sus alumnos como ciudadanos éticos, atentos y comprometidos con las transformaciones sociales y políticas de la sociedad; es decir, entendemos que, desde esta perspectiva, los profesores de Matemáticas deben formarse como profesionales democráticos.

Formar a los profesores de Matemáticas como profesionales democráticos, por fin, requiere la discusión de políticas públicas para la formación de profesores que tomen en cuenta los principios fundamentales para la construcción de la profesionalidad docente (Contreras, 2002), especialmente en el aspecto de entender la escuela como lugar de preparación para la vida futura de los alumnos basada en valores como la ciudadanía y la ética. 


\section{Consideraciones Finales}

La formación inicial de profesores es un proceso complejo y multidimensional que implica enormes desafíos que deben ser afrontados de manera articulada y conjunta por los formadores de profesores, las instituciones de formación y los organismos responsables de las políticas públicas educativas.

A su vez, formar a profesores de Matemáticas como profesionales democráticos hoy parece ser un camino largo y arduo, ya que nuestra experiencia como investigadores y formadores demuestra que las prácticas supervisadas no se valoran adecuadamente como momentos imprescindibles para construir una competencia profesional diferenciada, las escuelas no son debidamente reconocidas como instancias centrales para la consolidación del compromiso con la comunidad escolar y las políticas públicas de formación de profesores no se discuten de manera representativa, teniendo en cuenta la vida futura de los alumnos basada en valores más humanos.

Cuando recordamos la concepción de la comunidad escolar con el fin de considerar no solo a los alumnos y profesores, sino también a las familias de los alumnos, las asociaciones comunitarias locales y los movimientos sociales, es entonces cuando el abismo para la consolidación de compromisos por parte de profesores y universidades parece profundizarse. Las relaciones universidad / escuela arrojan algo de luz al final del túnel si fuéramos capaces, de manera cualitativa, de transformar las oportunidades que se han presentado para repensar nuestra "cultura académica" de formación inicial de profesores de Matemáticas.

El primer paso, probablemente el más difícil, es reconocer humildemente que, como señala Zeichner (2019), de poco sirve solo idealizar la formación de profesores de Matemáticas bajo la "perspectiva teórica de la justicia social" y, sin embargo, en la práctica, en detrimento del "conocimiento y experiencia que existe en las familias y comunidades de los alumnos", apostar a que solo el conocimiento matemático académico es capaz de modificar la vida de los alumnos. Transponiéndolo al contexto institucional, es urgente preguntarse si la universidad puede, de hecho, "salvar" la escuela en base a una relación jerárquica que desprecia / devalúa la historia de vida de toda la comunidad escolar.

En esta perspectiva, también es necesario contextualizar la viabilidad de construir alianzas entre los futuros profesores de Matemáticas, sus formadores y agentes de la comunidad escolar, premisa de la dialéctica del profesionalismo democrático. Las prácticas colaborativas 
evidenciadas en las acciones de extensión dejan una gama de posibilidades, ya que están ancladas en la tríada universitaria enseñanza-investigación-extensión. Institucionalmente, nos corresponde promover acciones de extensión que involucren de manera integral a la comunidad escolar, a partir de propuestas etnomatemáticas (D'Ambrosio, 2020) y, sin embargo, reconocer su contribución al proceso de formación de profesores, en la misma medida que la enseñanza y la investigación.

No obstante, el profesionalismo democrático exigirá mucho más de los investigadores, formadores e instituciones que forman profesores de Matemáticas. Ciertamente, será necesario repensar la profesionalidad docente que, como defendemos, debe centrarse en la construcción de una sociedad basada en los ideales de libertad, igualdad y, finalmente, justicia social.

\section{Referencias}

Barbosa, M. V. \& Fernandes, N. A. M. (2017). Políticas públicas para formação de professores: PIBID, mestrados profissionais e PNEM. En M. V. Barbosa \& N. A. M. Fernandes (Orgs.). Políticas públicas para a formação de professores (pp. 23-39). Brasília: Instituto Nacional de Estudos e Pesquisas Educacionais Anísio Teixeira.

Born, B. B.; Prado, A. P. \& Felippe, J. M. F. G. (2019). Profissionalismo docente e estratégias para o seu fortalecimento. Educação e Pesquisa, São Paulo, 45, e201945002003, 1-22. http://dx.doi.org/10.1590/S1678-4634201945002003

BRASIL (2000). Diretrizes Curriculares Nacionais para a Formação de Professores da Educação Básica. Brasília: Ministério da Educação / Conselho Nacional de Educação.

BRASIL (2001). Diretrizes Curriculares Nacionais para os Cursos de Matemática, Bacharelado e Licenciatura. Brasília: Ministério da Educação / Conselho Nacional de Educação.

Cyrino, M. C. C. T. (2013). Formação de professores que ensinam Matemática em comunidades de prática. Actas del VII Congreso Iberoamericano de Educación Matemática (pp. 51995206). Montevideo: S.E.M.UR.

Cyrino, M. C. C. T. (Org.) (2018). Temáticas emergentes de pesquisas sobre a formação de professores que ensinam Matemática: desafios e perspectivas. Brasília: Sociedade Brasileira de Educação Matemática. 1 ed.

Contreras, J. (2002). A autonomia de professores. São Paulo: Cortez. 1 ed.

Cuban, L. (1969). Teacher and community. Harvard Educational Review, Cambridge, MA, 39 (2), 253-272. http://doi.org/10.17763/haer.39.2. n7614t6x509x13n1

D'Ambrosio, U. (2020). Ethnomathematics: past and future. Revemop. Revista de Educação Matemática de Ouro Preto, Ouro Preto, 2, e202002, 1-14. http://doi.org/10.33532/revemop. $\underline{\mathrm{e} 202002}$ 
Dzur, A. W. (2008). Democratic professionalism: Citizen participation and the reconstruction of professional ethics, identity, and practice. University Park: The Pennsylvania State University Press.

Flores, M. A. (2014). Discursos do profissionalismo docente: paradoxos e alternativas conceituais. Revista Brasileira de Educação, Rio de Janeiro, 19 (59), 851-869. http://dx.doi.org/10.1590/S1413-24782014000900003

Franchi, R. H. O. L. \& Reis, F. S. (2012). The development of Mathematical Modelling activities from thematic projects in the social-critical perspective. Journal of Mathematics and System Science, Wilmington, 2, 341-347.

Freitas, M. A. (2004). Educação e ensino de língua estrangeira hoje: implicações para a formação de seus respectivos profissionais e aprendizes. En M. H. V. Abrahão (Org.). Prática de ensino de língua estrangeira: experiências e reflexões (pp. 117-130). Campinas: Pontes.

Feiman-Nemser, S. (2012). Teachers as Learners. Cambridge, MA: Harvard Education Press.

Fiorentini, D. (2009). Educação Matemática: diálogos entre universidade e escola. En Encontro Gaúcho de Educação Matemática, 10, 2009, Ijuí. Anais... Ijuí: UNIJUÍ, pp. 1-12.

Gastic, B. (2014). Closing the opportunity gap: Preparing the next generation of effective teachers. In F. M. Hess \& M. Q. McShane (Eds.). Teacher quality 2.0: Toward a new era in education reform (pp. 91-108). Cambridge, MA: Harvard Education Press.

Gewirtz, S.; Mahony, P.; Hextall, I. \& Cribb, A. (Eds.). (2009). Changing teacher professionalism: International trends, challenges and ways forward. New York and London: Routledge.

Hargreaves, D. (1994). The new professionalism: the synthesis of professional and institutional development. Teaching and Teacher Education, London, 10 (4), 423-438.

Nacarato, A. M. \& Paiva, M. A. V. (Orgs.) (2017). A formação do professor que ensina Matemática: perspectivas e pesquisas. Belo Horizonte: Autêntica. 1 ed.

Perrenoud, P. (2001). A prática reflexiva no oficio do professor: profissionalização e razão pedagógica. Porto Alegre: Artmed, 2002. 1 ed.

Pimenta, S. G. (1999). Saberes pedagógicos e atividade docente. São Paulo: Cortez. 1 ed.

Reis, F. S. \& Esteves, F. R. (2020). Contribuições das Tecnologias da Informação e Comunicação à formação de professores de Matemática na modalidade a distância. Revemop. Revista de Educação Matemática de Ouro Preto, Ouro Preto, 2, e202020, 1-21. http://doi.org/10.33532/revemop. e202020

Rosa, M.; Reis, F. S. \& Orey, D. C. (2012). A Modelagem Matemática Crítica nos cursos de formação de professores de Matemática. Acta Scientiae, Canoas, 14, 159-184.

Sachs, J. (2000). The activist professional. Journal of Educational Change, Dordrecht, 1 (1), 77-95.

Sachs, J. (2001). Teacher professional identity: Competing discourses, competing outcomes. Journal of Education Policy, London, 16 (2), 149-161. http://doi.org/10.1080/02680930116819 
Sachs, J. (2003). The activist teaching profession. Buckingham: Open Unversity Press.

Santana, F. C. M. \& Barbosa, J. C. (2019). A relação universidade / escola e o Programa Residência Pedagógica / Subprojeto de Matemática: estratégias de poder e modos de subjetivação. ReviSeM. Revista Sergipana de Matemática e Educação Matemática, Aracaju, 2, 1-24. http://doi.org/10.34179/revisem.v4i2.11755

Sockett, H. (1993). The moral base for teacher professionalism. Columbia: Teacher's College Press.

Vargas, S. G. (2017). Políticas públicas relacionadas con la formación de profesores en Costa Rica. In M. V. Barbosa \& N. A. M. Fernandes (Orgs.). Políticas públicas para a formação de professores (pp. 151-168). Brasília: Instituto Nacional de Estudos e Pesquisas Educacionais Anísio Teixeira.

Zeichner, K. (2009). Teacher education and the struggle for social justice. New York: Routledge.

Zeichner, K. (2016). Independent teacher education programs: Apocryphal claims, illusory evidence. Boulder: National Education Policy Center and University of Colorado School of Education.

Zeichner, K. (2018). The struggle for the soul of teacher education. New York: Routledge.

Zeichner, K. (2019). Preparing Teachers as Democratic Professionals. Action in Teacher Education, London, 42, 38-48. https://doi.org/10.1080/01626620.2019.1700847 